\title{
Evaluación económica del tratamiento antimicrobiano en pacientes histerectomizadas por fibroma uterino
}

\author{
Katherine Romero V. MSc ${ }^{1 a}$, Irayma Cazull I. PhD. ${ }^{2}$, Lilian González L. $3 b$ \\ ${ }^{1}$ Facultad de Ciencias Médicas, Universidad Regional Autónoma de los Andes, Ambato, Ecuador. ${ }^{2}$ Servicio de Auditoría \\ Médica, Servicio de Medicina Interna; ${ }^{3}$ Departamento de Economía y Contabilidad. Hospital General Docente Dr. Agostinho \\ Neto, Guantánamo, Cuba. \\ $a_{\text {Licenciada en Ciencias Farmacéuticas. }}{ }^{\mathrm{b}}$ Licenciada en Economía de la Salud.
}

\section{RESUMEN}

Antecedentes: Las infecciones constituyen la principal causa de morbilidad luego de las cirugías ginecológicas. Debido al uso irracional de los antimicrobianos y la poca adherencia a los protocolos de actuación, se decidió investigar la alternativa clínica más eficiente entre las existentes para alcanzar un objetivo sanitariamente deseable. Objetivos: Realizar una evaluación económica del uso de los antimicrobianos en las pacientes operadas con diagnóstico de fibroma uterino en el Hospital "Agostinho Neto", Guantánamo, Cuba, durante el periodo abril-mayo de 2012 para lograr un uso racional de estos medicamentos, disminuir riesgo de resistencia antimicrobiana, incidencia de infección de la herida quirúrgica y costos por concepto de medicamentos. Método: Estudio retrospectivo, en base a revisión de historias clínicas, se determinó la edad de las pacientes, tipo de cirugía, antimicrobianos más utilizados y duración del tratamiento. Se realizó una evaluación económica total del tipo minimización de costos. Resultados: El 61,64\% de las pacientes tenían entre $41-50$ años. El $81,62 \%$ de las cirugías se clasificó como limpia contaminada; el antimicrobiano más utilizado fue la cefazolina como monoterapia (51,28\%); el $84,62 \%$ de las pacientes recibió tratamiento durante 2 días; el $100 \%$ de las prescripciones resultó inadecuada; el costo global por concepto de antimicrobianos fue de $3.242,65$ pesos cubanos y se hubiese obtenido un ahorro aproximado de 827,80 pesos cubanos de haberse aplicado correctamente las protocolos de actuación. Conclusión: Los profesionales del servicio de ginecología no se adhieren a los protocolos de profilaxis antibiótica preoperatoria, aumentando los costos por medicamentos.

\section{PALABRAS CLAVE: Farmacoeconomía, histerectomía, profilaxis antibiótica preoperatoria, Cuba}

\section{SUMMARY}

Background: Infections are the main cause of morbidity after gynecological surgeries. Due to the irrational use of antimicrobials and little adherence to protocols, it decided to investigate the most efficient clinical alternative among existing sanitary desirable to reach a goal. Aims: To perform an economic evaluation of the use of antimicrobials in patients operated with a diagnosis of uterine fibroid in the "Agostinho Neto" Hospital, Guantanamo, Cuba, during the period April-May 2012 to achieve a rational use of these drugs reduce risk of antimicrobial resistance, incidence of surgical wound infection and costs for drugs. Method: A retrospective study was made, the medical records were reviewed, the patient age, type of surgery, most commonly used antimicrobial and duration of treatment was determined: an overall economic assessment, the type was minimization of costs. Results: $61.64 \%$ of the patients were between $41-50$ years old; the $81.62 \%$ of the surgeries were classified as clean contaminated; cefazolin was the most antimicrobial used as monotherapy $(51.28 \%) ; 84.62 \%$ of the patients received treatment for 2 days; $100 \%$ of prescriptions was inadequate; 
the overall cost was 3,242.65 Cuban pesos and has been obtained savings of approximately 728.80 Cuban pesos if the protocols have been correctly applied. Conclusion: Gynecology service professionals do not adhere to the protocols of preoperative antibiotic prophylaxis, increasing drug cost.

\section{KEY WORDS: Pharmacoeconomics, hysterectomy, preoperative antibiotic prophylaxis, Cuba}

\section{INTRODUCCIÓN}

La farmacoeconomía, puede definirse como la aplicación del análisis económico al campo de los medicamentos $(1,2)$. Su aplicación práctica se basa en que el paciente debe recibir la atención que requiere para mantener su salud pero a través de estudios que permitan respetar ese principio mediante la eficiencia y racionalidad en el uso de los fármacos (3).

Se reconocen distintos métodos de evaluación económica. Michael F. Drummond ideó una clasificación muy interesante donde los métodos de evaluación farmacoeconómica se dividen en parciales y completos atendiendo a: costos, consecuencias o resultados y alternativas. Si se prescinde de uno o más de estos tres elementos, el estudio se clasifica como parcial y se plantea que no sirve para medir eficiencia (4).

El constante perfeccionamiento que persigue la práctica sanitaria en Cuba, unida a la difícil situación económica imperante, impone cada vez con más fuerza la necesidad de reducir las decisiones arbitrarias en la utilización de los escasos recursos disponibles. Esto implica la necesidad de aplicar instrumentos que perfeccionen los mecanismos de toma de decisiones que redunden en mejores resultados sanitarios con costos prudenciales para la sociedad. La evaluación económica de los medicamentos no resuelve todos los problemas que enfrentan diariamente los profesionales de la salud, pero si podría ser uno de estos instrumentos de gran utilidad (4-7).

Dentro del campo de la salud, las infecciones constituyen la principal causa de morbilidad luego de los procedimientos quirúrgicos, siendo la histerectomía y la cesárea segmentaria las operaciones más frecuentes en ginecología (8). Al analizar todas estas consideraciones, sumado a que aún los medicamentos se utilizan de forma irracional y que existe poca adherencia a los protocolos de actuación, los autores del trabajo se preguntan: ¿Cuál es la alternativa clínica más eficiente entre las existentes para alcanzar un objetivo sanitariamente deseable?

Ante esta interrogante se decide realizar una evaluación económica del uso de los antimicrobianos en las pacientes operadas con el diagnóstico de fibroma uterino en el Hospital General Docente "Dr. Agostinho Neto" de Guantánamo, Cuba, durante el periodo abril-mayo de 2012 con el objetivo de lograr un uso racional de este tipo de medicamentos, disminuyendo así, el riesgo de resistencia antimicrobiana, la incidencia de infección de la herida quirúrgica y los costos por concepto de medicamentos.

\section{PACIENTES Y MÉTODO}

Se realizó un estudio retrospectivo de las pacientes histerectomizadas con el diagnóstico de fibroma uterino en el Hospital General Docente "Dr. Agostinho Neto" de Guantánamo, Cuba, durante el periodo abril-mayo de 2012, con el objetivo de realizar una evaluación económica, del tipo minimización de costos, del tratamiento utilizado. Los datos fueron obtenidos a partir de la revisión de las historias clínicas de las pacientes programadas para cirugía en el servicio de ginecología de dicha institución durante el periodo de estudio.

La población se conformó por todas las pacientes que ingresaron con el diagnóstico de fibroma uterino sintomático y que se les realizó histerectomía total abdominal. Fueron excluidas aquellas que no fueron intervenidas quirúrgicamente y que solo se les realizó legrado diagnóstico. El tamaño de muestra determinado fue de 117 pacientes.

Se constató la existencia del informe operatorio donde se especifica la clasificación de la cirugía mediante las siguientes variables:

Limpia: aquella en la cual se mantiene una técnica aséptica, no hay inflamación y no se compromete el tracto genitourinario, gastrointestinal ni respiratorio. Tales operaciones son usualmente electivas y la herida se cicatriza casi siempre en primera intención. El riesgo de infección es menor al $5 \%$. No requiere profilaxis antibiótica preoperatoria salvo en pacientes inmunocomprometidos, cirugía con implantes o mayores de 65 años.

Limpia-contaminada: compromete al tracto genitourinario, gastrointestinal o respiratorio pero la contaminación con su contenido no es significativa. El riesgo de infección es del 10\%. Requiere profilaxis antibiótica preoperatoria.

Contaminada: aquella en la cual se encuentra inflamación aguda sin exudado, incluyen aquellas cirugías donde se ha roto la técnica aséptica o volcado contenido de una víscera hueca. El riesgo de infección es del $20 \%$. Requiere profilaxis antibiótica preoperatoria. 
Sucia: incluye aquellas sobre heridas inveteradas, con abscesos, material purulento o una víscera perforada. La tasa de infección excede el $30 \%$. Requiere tratamiento antimicrobiano empírico $(9,10,11)$.

En el servicio objeto de estudio, se encuentra diseñado el protocolo para las cirugías ginecológicas según la clasificación mostrada anteriormente y atendiendo a las normas nacionales e internacionales (12), quedando establecido la cefazolina $2 \mathrm{~g}$ EV en el pre-operatorio (de 20-40 minutos previo a la cirugía) y en caso de alergia a las cefalosporinas, administrar gentamicina $80 \mathrm{mg}$ EV en el pre-operatorio (de 20-40 minutos previo a la cirugía).

Se consideró las observaciones directas y anotaciones que en el expediente clínico hicieron los médicos tratantes durante la estadía hospitalaria.

El uso de los antimicrobianos se analizó según la clasificación de la cirugía donde se empleó. Para ello se diseñó un modelo de recogida de datos en el cual se reflejaron los datos personales de la paciente, tipo de cirugía, antimicrobianos utilizados y duración del tratamiento.

Para evaluar la calidad de la prescripción se establecieron dos variables: i. Adecuada: aquella que se ajusta a los protocolo de antibióticos institucional según el tipo de cirugía. ii. Inadecuada: aquella que no se ajusta a los protocolos de antibióticos institucional según el tipo de cirugía.

Según la clasificación de los distintos métodos de evaluación económica realizada por Michael F. Drummond, los estudios completos de evaluación económica son los siguientes (4):

Estudios de costo-beneficio: la característica principal es que tanto los costos como los efectos de las opciones comparadas se miden en unidades monetarias. Desde el punto de vista económico se considera la forma de análisis más ortodoxa.

Estudios de costo-efectividad: se comparan los efectos de las distintas opciones en unidades clínicas, entre las cuales se encuentran, por ejemplo, los años de vida ganados, los ingresos hospitalarios evitados, el número de casos prevenidos, las vidas salvadas y las complicaciones evitadas. Los resultados pueden presentarse como valor medio de la razón de costos a efectividad, o bien como incremento de dicha razón (costo-efectividad incremental).

Estudios de costo-utilidad: buscan valorar la vida ganada no solo en términos de cantidad (años de vida), sino también de calidad. Las unidades que se obtienen son los años de vida ajustados por calidad (AVAC) y los resultados se presentan de la misma forma que en el análisis de costo-efectividad, pero usando como denominador de la fracción los AVAC obtenidos en lugar de la efectividad.

Análisis de minimización de costos: se utilizan cuando no hay diferencia entre los efectos de las alternativas comparadas. En este caso se selecciona la opción menos costosa. Siendo este último el utilizado en nuestra investigación.

Para dar cumplimiento al próximo objetivo, se calculó el costo global de los antimicrobianos utilizados (costo global real), obteniéndose el dato de la hoja de las indicaciones médicas de las historias clínicas de las pacientes estudiadas, los mismos se consignaron en las fichas con su nombre genérico, se sumaron las dosis empleadas en cada paciente y se anotaron: las dosis globales, el costo por unidad y el costo global, para obtener finalmente la suma en valor de todos los antimicrobianos en el periodo de estudio.

Se determinó además, a través del mismo procedimiento, el costo global que se hubiese obtenido por concepto de esta terapéutica en este tipo de pacientes (costo global teórico) atendiendo al tratamiento que realmente debía haberse impuesto según protocolo de actuación para cada tipo de cirugía.

El ahorro se determinó a partir de la diferencia existente entre el costo global real menos el costo global teórico.

Los valores fueron expresados en Moneda Nacional (MN) y posteriormente en dólares americanos (USD), para ello, en primer lugar, hubo de llevar los valores en MN a pesos cubanos convertibles (CUC) con un valor en el país de 25 pesos cubanos para obtener 1 CUC y estos a su vez convertirlos en USD, actualmente en Cuba con un valor de 0,86 centavos de dólar, por lo que el equivalente en moneda americana se multiplica por el valor de CUC obtenido.

Todos los resultados se evaluaron mediante la determinación de valores porcentuales para cada una de las variables.

\section{RESULTADOS}

En las pacientes operadas de fibroma uterino según edad durante el periodo de estudio, se observó un predominio de las mujeres en las edades comprendidas entre los 41 y 50 años $(61,54 \%)$, seguida de las pacientes con edades comprendidas entre los 31 y 40 años $(19,66 \%)$ y un menor porcentaje de pacientes en las edades extremas de la adultez (Tabla I).

\section{Tabla I}

PACIENTES HISTERECTOMIZADAS SEGÚN EDAD

\begin{tabular}{ccc}
\hline $\begin{array}{c}\text { Grupo de edades } \\
\text { (años) }\end{array}$ & $\mathrm{n}$ & $\%$ \\
\hline $20-30$ & 9 & 7,69 \\
$31-40$ & 23 & 19,66 \\
$41-50$ & 72 & 61,54 \\
$51-60$ & 13 & 11,11 \\
\hline Total & 117 & 100 \\
\hline
\end{tabular}


Según la clasificación de las cirugías, predominaron las cirugías limpia-contaminada $(84,62 \%)$, seguida de las cirugías sucias $(10,26 \%)$, contaminadas $(4,27 \%)$ y cirugía limpia $(0,85 \%)$.

En la Tabla II se muestran los diferentes esquemas de tratamiento antimicrobiano que se utilizaron según el tipo de cirugía, existiendo un predominio del uso de la cefazolina como monoterapia $(51,28 \%)$, a la cual le sigue la combinación de este medicamento con gentamicina y metronidazol $(23,07 \%)$, y la combinación con gentamicina solamente $(10,26 \%)$, por lo que el uso de este antibiótico se hace mucho mayor.

En la cirugía limpia-contaminada, se observa igualmente que la cefazolina como monoterapia fue la que más se usó comparada con el resto de los esquemas de tratamiento $(48,72 \%)$, seguido de la combinación de este medicamento con gentamicina y metronidazol $(15,38 \%)$. Los esquemas de tratamiento menos utilizados en este tipo de cirugía fueron las combinaciones penicilina cristalina + gentamicina + metronidazol $(3,42 \%)$, y cefazolina + metronidazol $(3,42 \%)$, también se usó la penicilina cristalina como monoterapia $(8,55 \%)$.
La Tabla III muestra que la mayoría de las pacientes operadas recibieron tratamiento antibiótico durante 2 días $(84,62 \%)$ y le siguen las que recibieron tratamiento durante 4 días $(10,26 \%), 3$ días $(4,27 \%)$ y 1 día $(0,85 \%)$.

No hubo efectos adversos del uso de antibióticos, en cualquiera de los esquemas usados, por lo que no se elevaron los costos por estadía hospitalaria ni por el uso de otros medicamentos para tratar las reacciones medicamentosas.

Como se muestra en la Tabla IV, para el tratamiento de las pacientes operadas por fibroma uterino sintomático durante el periodo de estudio, se utilizaron un total de 1.572 unidades de antibióticos, distribuidos en 4 tipos. La prescripción mayoritaria la constituyó la cefazolina $(45,93 \%)$, seguida por gentamicina $(18,38 \%)$, penicilina cristalina $(18,32 \%)$ y el metronidazol $(17,37 \%)$.

El costo global por concepto de uso de este tipo de medicamentos en las pacientes estudiadas fue de 3.242,65 MN. La diferencia entre costo global real $(3.242,65 \mathrm{MN})$ y costo global teórico $(2.414,85$ $\mathrm{MN}$ ) fue de $827,80 \mathrm{MN}$, siendo su equivalente en USD un valor de 28,47 .

\section{Tabla II \\ ESQUEMAS DE TRATAMIENTO ANTIMICROBIANO ENDOVENOSO QUE SE UTILIZAN SEGÚN EL TIPO DE CIRUGÍA}

\begin{tabular}{|c|c|c|c|c|c|c|c|c|c|c|}
\hline \multirow[t]{3}{*}{ Antimicrobianos } & \multicolumn{10}{|c|}{ Tipo de cirugía } \\
\hline & \multicolumn{2}{|c|}{ Limpia } & \multicolumn{2}{|c|}{$\begin{array}{c}\text { Limpia } \\
\text { contaminada }\end{array}$} & \multicolumn{2}{|c|}{ Contaminada } & \multicolumn{2}{|c|}{ Sucia } & \multicolumn{2}{|c|}{ Total } \\
\hline & $\mathrm{n}$ & $\%$ & $\mathrm{n}$ & $\%$ & $\mathrm{n}$ & $\%$ & $\mathrm{n}$ & $\%$ & $\mathrm{n}$ & $\%$ \\
\hline $\begin{array}{c}\text { Penicilina cristalina } \\
1000000 \mathrm{UI}\end{array}$ & - & - & 10 & 8,55 & - & - & - & - & 10 & 8,55 \\
\hline $\begin{array}{c}\text { Penicilina cristalina } \\
+ \\
\begin{array}{c}+ \\
\text { Gentamicina } 80 \mathrm{mg} \\
+ \\
\text { Metronidazol } 250 \mathrm{mg}\end{array}\end{array}$ & - & - & 4 & 3,42 & - & - & - & - & 4 & 3,42 \\
\hline Cefazolina $1 \mathrm{~g}$ & 1 & 0,85 & 57 & 48,72 & - & - & 2 & 1,71 & 60 & 51,28 \\
\hline $\begin{array}{c}\text { Cefazolina } 1 \mathrm{~g} \\
\stackrel{+}{+} \\
\text { Gentamicina } 80 \mathrm{mg}\end{array}$ & - & - & 10 & 8,55 & - & - & 2 & 1,71 & 12 & 10,26 \\
\hline $\begin{array}{c}\text { Cefazolina } 1 \mathrm{~g} \\
+ \\
\text { Metronidazol } 500 \mathrm{mg}\end{array}$ & - & - & - & - & - & - & 4 & 3,42 & 4 & 3,42 \\
\hline $\begin{array}{c}\text { Cefazolina } 1 \mathrm{~g} \\
+ \\
\text { Gentamicina } 80 \mathrm{mg} \\
+ \\
\text { Metronidazol } 500 \mathrm{mg}\end{array}$ & - & - & 18 & 15,38 & 5 & 4,27 & 4 & 3,42 & 27 & 23,07 \\
\hline
\end{tabular}

Total

$1 \quad 0,85$

$99 \quad 84,62$

$5 \quad 4,27$

1210,26

117100 


\section{DURACIÓN DEL TRATAMIENTO ANTIMICROBIANO}

\begin{tabular}{|c|c|c|c|c|c|c|c|c|c|c|}
\hline \multirow[t]{3}{*}{ Antimicrobianos } & \multicolumn{10}{|c|}{ Tiempo de tratamiento } \\
\hline & \multicolumn{2}{|c|}{1 día } & \multicolumn{2}{|c|}{2 días } & \multicolumn{2}{|c|}{3 días } & \multicolumn{2}{|c|}{4 días } & \multicolumn{2}{|c|}{ Total } \\
\hline & $\mathrm{n}$ & $\%$ & $\mathrm{n}$ & $\%$ & $\mathrm{n}$ & $\%$ & $\mathrm{n}$ & $\%$ & $\mathrm{n}$ & $\%$ \\
\hline $\begin{array}{c}\text { Penicilina cristalina } \\
1000000 \mathrm{UI}\end{array}$ & - & - & 10 & 8,55 & - & - & - & - & 10 & 8,55 \\
\hline $\begin{array}{c}\text { Penicilina cristalina } \\
++ \\
\text { Gentamicina } 80 \mathrm{mg} \\
+ \\
\text { Metronidazol } 500 \mathrm{mg}\end{array}$ & - & - & 4 & 3,42 & - & - & - & - & 4 & 3,42 \\
\hline Cefazolina $1 \mathrm{~g}$ & 1 & 0,85 & 57 & 48,72 & - & - & 2 & 1,71 & 60 & 51,28 \\
\hline $\begin{array}{c}\text { Cefazolina } 1 \mathrm{~g} \\
+\underset{+}{\text { Gentamicina } 80 \mathrm{mg}}\end{array}$ & - & - & 10 & 8,55 & - & - & 2 & 1,71 & 12 & 10,26 \\
\hline $\begin{array}{c}\text { Cefazolina } 1 \mathrm{~g} \\
+ \\
\text { Metronidazol } 500 \mathrm{mg}\end{array}$ & - & - & - & - & - & - & 4 & 3,42 & 4 & 3,42 \\
\hline $\begin{array}{c}\text { Cefazolina } 1 \mathrm{~g} \\
++ \\
\text { Gentamicina } 80 \mathrm{mg} \\
+ \\
\text { Metronidazol } 500 \mathrm{mg}\end{array}$ & - & - & 18 & 15,38 & 5 & 4,27 & 4 & 3,42 & 27 & 23,07 \\
\hline Total & 1 & 0,85 & 99 & 84,62 & 5 & 4,27 & 12 & 10,26 & 117 & 100 \\
\hline
\end{tabular}

Tabla IV

COSTO GLOBAL DE LOS ANTIMICROBIANOS EMPLEADOS

\begin{tabular}{lccccc}
\hline \multicolumn{1}{c}{ Antimicrobianos } & \multicolumn{2}{c}{$\begin{array}{c}\text { Forma farmacéutica } \\
\text { (U) }\end{array}$} & $\begin{array}{l}\text { Costo por unidad } \\
(\mathrm{MN})\end{array}$ & $\begin{array}{c}\text { Costo global } \\
(\mathrm{MN})\end{array}$ & $\begin{array}{c}\text { Costo global } \\
(\text { USD) }\end{array}$ \\
\hline $\begin{array}{l}\text { Penicilina Cristalina } \\
1 \text { 000 000 UI }\end{array}$ & 288 & 18,32 & 0,35 & 100,8 & 3,47 \\
Cefazolina 1g & 722 & 45,93 & 2,30 & 1660,6 & 57,12 \\
Gentamicina 80 mg & 289 & 18,38 & 3,00 & 867 & 29,82 \\
Metronidazol 500 mg & 273 & 17,37 & 2,25 & 614,25 & 21,13 \\
\hline Costo global & 1.572 & 100 & 7,90 & $3.242,65$ & 111,54 \\
\hline
\end{tabular}

\section{DISCUSIÓN}

Los resultados obtenidos en nuestra investigación relacionados la selección del medicamento, dosis a utilizar y tiempo de tratamiento, muestran que la prescripción de los medicamentos en la profilaxis antibiótica preoperatoria en la histerectomía electiva por fibroma uterino sintomático fue inadecuado en todos los casos, determinando un aumento del costo por concepto de medicamentos.

Los miomas o fibromas, son tumores benignos que aparecen frecuentemente en el útero, y aunque a veces pueden ser asintomáticos, en muchas mujeres son la razón más común de las intervenciones ginecológicas (13). 
Es uno de los diagnósticos más comunes en las pacientes intervenidas quirúrgicamente en los servicios de ginecología y aparecen, fundamentalmente, entre los 30 y 50 años de edad, aunque en la actualidad se diagnostica más temprano por el uso tan difundido de la ultrasonografía, lo que permite el diagnóstico de tumores muy pequeños, antes de que sean capaces de producir síntomas, resultados que coinciden con los obtenidos en esta investigación $(14,15)$.

Sobre la base del grado esperado de contaminación microbiana, históricamente, los cirujanos y epidemiólogos han estratificado los procedimientos quirúrgicos en: limpios, limpios-contaminados, contaminados y sucios. En el estudio se identificó una cirugía limpia, dato con el cual los autores no están de acuerdo pues conceptualmente se contradice ya que la histerectomía incluye el tracto genital, de haber sido así, en este caso tampoco debió utilizarse la cefazolina cuando la literatura plantea que en estos casos no se requiere de profilaxis antibiótica.

En las cirugías clasificadas como limpia-contaminada, no era necesario la administración de otros antimicrobianos, como la gentamicina y el metronidazol para combinar la terapia, según refiere la literatura para este tipo de cirugía, estudios realizados no demostraron diferencias significativas en cuanto al efecto logrado utilizando cefalosporina como monoterapia y combinada con metronidazol (16). Con relación a la dosis, tampoco se encontraron resultados concordantes con la literatura, pues en ninguno de los casos se utilizó como dosis única, sino cada 8 horas y por varios días.

La utilización de antibióticos en la prevención de la infección de la herida ha suscitado controversias e informaciones contradictorias. Hoy día está demostrada la eficacia de la profilaxis perioperatoria en situaciones concretas y están bien establecidas las indicaciones y técnicas de su utilización (17).

El agente antiinfeccioso que se escoja debe ser activo frente a los posibles microorganismos que van a provocar el proceso infeccioso postquirúrgico e incluye, los habituales en el territorio a intervenir y los aportados del exterior. En general las cefalosporinas son los fármacos más empleados en profilaxis quirúrgica, debido a su espectro antibacteriano y su baja incidencia de reacciones alérgicas y efectos secundarios $(17,18,19)$.

En ginecología las infecciones suelen deberse a Stafilococcus, Streptococcus, Enterococcus, E. coli, Bacteroides, Fusobacterium, y se considera idónea la cefazolina. Su vida media es moderadamente prolongada (1,8 horas) y su espectro antibacteriano incluye $S$. aureus, otros cocos gram (+) exceptuando enterococo, bacilos gram (-) comunes aerobios, y anaerobios gram (+) y (-), aunque no activo frente a $\mathrm{C}$. difficile y $\mathrm{B}$. fragilis. Se administrará vía intravenosa inmediatamente antes de la intervención en la inducción anestésica o al menos dentro de la hora previa al inicio de la cirugía. Si es por vía intramuscular u oral, se administrará 1 hora antes. Para pacientes con historia de alergia a $\beta$-lactámicos, el metronidazol con o sin aminoglucósido es una buena alternativa $(17,20)$.

Lamentablemente, los resultados que se obtuvieron en la investigación no coincidieron con los aportados por la literatura y otros estudios realizados $(17,21)$.

Con relación a los esquemas de tratamiento utilizados en las cirugías contaminadas y sucias, también se presencia dificultad con la prescripción pues en estos casos es de suponer que exista una flora polimicrobiana donde, a pesar de que debe aplicarse terapia con varios antimicrobianos, la cefazolina no debe ser utilizada por dos razones fundamentales: su menor espectro de acción comparada con las cefalosporinas de 3a generación y que los medicamentos utilizados como profilaxis no deben ser indicados como tratamiento para la misma patología.

Existen pocas evidencias que sostienen el uso de antimicrobianos pasada la cirugía, se plantea que el período efectivo no es mayor de 3 horas luego de la contaminación tisular, y que la contaminación bacteriana suele terminar cuando se produce el cierre de la herida, por lo que continuar la administración del antibiótico luego de cerrada la piel no supone beneficio alguno $(17,22,23,24)$.

Este hecho se verifica en la práctica, y ya numerosos trabajos prueban la utilidad de una única dosis de antimicrobiano administrado justo previo a la cirugía en operaciones del tracto biliar, cirugía gástrica, histerectomía y cesárea (25).

En este caso, los resultados arrojados por nuestro estudio tampoco coinciden con lo planteado en la bibliografía, pues en ninguno de los casos donde se podía utilizar la profilaxis antibiótica preoperatoria se hizo y estas pacientes se mantuvieron varios días con tratamiento, con el consiguiente aumento de riesgo de aparición de reacciones adversas, resistencia antimicrobiana, aumento de la estadía hospitalaria y de los costos por concepto de medicamentos.

Bien es cierto que existen pacientes con mayor riesgo de infección, como es el caso de pacientes inmunocomprometidas, obesas, diabéticas o con otros factores predisponentes, pero la muestra objeto de estudio no guarda relación con dicho planteamiento, por lo que no existe justificación para el empleo de otro esquema de tratamiento ajeno al protocolo establecido.

Dado que este trabajo demostró que en el Hospital General Docente Dr. Agostinho Neto no se realiza la profilaxis antibiótica preoperatoria en la histerectomía total abdominal, sino que se les impone tratamiento antibiótico a las pacientes, no se pudo realizar una comparación costo-efectividad respecto al tema, por lo que la determinación del 
costo global por este concepto se realizó a partir del supuesto tratamiento si se hubiese aplicado adecuadamente la profilaxis preoperatoria (cefazolina en monodosis o gentamicina en el caso de alergia a los $\beta$ lactámicos), obteniéndose en este caso un valor de 2.414,85 $\mathrm{MN}$, derivándose un ahorro de $827,80 \mathrm{MN}$. Resultados similares fueron reportados en investigaciones internacionales relacionadas con el tema, considerándose como posible causa fundamental la formación e incorporación constante de nuevos médicos al servicio y la insuficiente socialización de los protocolos diseñados $(26,27,28)$.

Al determinar la equivalencia a USD, se podría haber obtenido un ahorro de 28,47 USD, valor que podría parecer irrelevante o provocar que los profesionales se despreocupen un tanto del tema, representando en la realidad todo lo contrario al obtenerse un efecto de bola de nieve si se toma en consideración que este valor es solo una parte de todo el proceder quirúrgico en ginecología y en un periodo de tiempo limitado.

Luego de un análisis exhaustivo de los datos obtenidos y según reportes de un sinnúmero de investigaciones, se puede plantear, que del grupo de medicamentos que se consumen en los hospitales, los antimicrobianos son los que se utilizan con mayores deficiencias $(29,30)$, ello, unido a su costo en sentido general, a su dependencia de la importación en algunos casos y al irremediable daño que produce en los enfermos su uso indiscriminado, justifican con creces el establecimiento de políticas de utilización efectivas conocidas como "Políticas de antimicrobianos".

\section{CONCLUSIÓN}

La prescripción de los medicamentos en la profilaxis antibiótica preoperatoria en la histerectomía electiva por fibroma uterino sintomático fue inadecuada en todos los casos, determinando un aumento del costo por concepto de medicamentos. Los profesionales muestran escasa adherencia a los protocolos establecidos de profilaxis antibiótica según tipo de cirugía.

\section{REFERENCIAS}

1. SOIKOS. Glosario de términos y conceptos de uso frecuente en la evaluación económica de medicamentos y programas sanitarios. Barcelona: Química Farmacéutica Bayer, 1996:11.

2. Collazo M, Rovira J. Criterios sobre la evaluación económica de los medicamentos antineoplásicos. Rev Cubana Oncol 1997;13(1):23-30.

3. Collazo M, Casademunt N. La farmacoeconomía en la industria farmacéutica y el sistema sanitario de Cuba. Rev Panam Salud Pública 2001;10(4):263-7.
4. Drummond MF, Sculpher MJ, Torrance GW, O'Brien BJ, Stoddard GL. Methods for the economic evaluation of health care programmes. Third edition. London: Oxford University Press 2005.

5. Del Prete S. Economía y salud en tiempos de reformas. Buenos Aires: Editorial Universidad Nacional de La Plata, 2000.

6. Badía X, Rovira J. Evaluación económica de medicamentos. Un instrumento para la toma de decisiones en la práctica clínica y la política sanitaria: Madrid Editorial Luzán S.S.A de Ediciones 1994:19.

7. Collazo MM. La aplicación práctica de la farmacoeconomía en la utilización de los medicamentos en Cuba. Rev Bras Cienc Farm 2004;40(4):539-48.

8. Stein GE. Patient costs for prophilaxis and treatment of obstetric and gynecologic surgical infections. Am J Obstet Gynecol 1991;164:1377-80.

9. Hemsell DL. Prophylactic antibiotics in gynecologic and obstetric surgery. J Infec Dis 1991;13(suppl 10):21-41.

10. CIRURED. (2007). Profilaxis antibiótica preoperatoria. Disponible en: http://www.sld.cu/uvs/cirured/temas.php?idv=6590.

11. Romero Sánchez RE, Landín Sorí M, García Rodríguez M. Actualidad en infecciones quirúrgicas. Revista Archivos de Medicina Camagüey 2012;16(4):15107. Disponible en: http://www.researchgate.net/ publication/262630841.

12. Van Eyk N, van Schalkwyk J, Infectious Diseases Committee. Antibiotic prophylaxis in gynaecologic procedures. J Obstet Gynaecol Can 2012;34(4):38291.

13. Cabezas E, Cutié E, Santiesteban S. Leiomioma uterino. En: Sánchez T (Eds). MANUAL DE PROCEDIMIENTOS EN GINECOLOGÍA: Editorial Ciencias Médicas, 2006; pp 88-9

14. Rigol O. Afecciones benignas del útero. En: Sánchez T (Eds). OBSTETRICIA Y GINECOLOGÍA: Editorial Ciencias Médicas 2004;293-5.

15. Aglietti $P$, Salvati EA, Wilson PD, Kutner LJ. Effect of a surgical horizontal unidireccional filtered air flow unit on wound bacterial contamination and wound healing. Clin Orthop Relat Res 1974;101:99-104.

16. Brummer TH, Heikkinen AM, Jalkanen J, Fraser J, Mäkinen J, Tomás E, et al. Antibiotic prophylaxis for hysterectomy, a prospective cohort study: cefuroxime, metronidazole, or both? BJOG 2013;120(10):126976.

17. Marcotti A. Profilaxis antibiótica en cirugía. Medwave 2001;1(05)e3087. Disponible en: URL: http://www.medwave.cl/link.cgi/Medwave/Cursos/ modll/3087?tab=metrica.

18. Gómez M, Martínez C, González B, Rubio MJ, Palomo JC, Quijada JL, et al. (2011). Guía Profilaxis Antimicrobiana Perioperatoria. GRUPO DE TRABAJO DE LA COMISION DE INFECCIONES Y POLITICA ANTIBIOTICA. Disponible en: URL: http://www.hvluz.es/ docs/Guia_Profilaxis_Antimicrobiana_Perioperatoria. pdf

19. Phoolcharoen N, Nilgate S, Rattanapuntamanee O, Limpongsanurak S, Chaithongwongwatthana S. A randomized controlled trial comparing ceftriaxone with cefazolin for antibiotic prophylaxis in abdominal hysterectomy. Int J Gynaecol Obstet 2012:119(1):11-3. 
20. Alfaro JM, Vicente M, Cruz Y, Pisonero JJ. Actualización sobre el uso de antimicrobianos en el Servicio de Cirugía General del Hospital Clínico Quirúrgico «Joaquín Albarrán». 2009 Disponible en: URL: http://www. bvs.sld.cu/revistas/cir/vol49_3_10/cir13310.pdf.

21. Mensa J, Gatell JM, Aznza JR, Domínguez-Gil A, García JE, Jimenez de Anta MT, Prats G. Profilaxis antibiótica en cirugía. Guía de terapéutica antimicrobiana. (18 ed Masson) 2008; pp 498-503.

22. Red de Hospitales Universitarios, Universidad de Buenos Aires y Sociedad Argentina de Infectología. Guía para la profilaxis antibiótica prequirúrgica. (2003) Disponible en: URL: http://consultorsalud. com/biblioteca/articulos/Guia\%20de\%20la\%20Profilaxis\%20quirugica\%20-\%20Sociedad\%20Argentina\%20de\%20Infectologia.pdf.

23. Kaiser $A B$. Antimicrobial prophylaxis in surgery. $N$ Engl J Med 1986;315:1129-38.

24. Su HY, Ding DC, Chen DC, Lu MF, Liu JY, Chang FY. Prospective randomized comparison of single-dose versus 1-day cefazolin for prophylaxis in gynecologic surgery. Acta Obstet Gynecol Scand 2005;84(4):3849.
25. DiPiro JT, Vallner JJ. Bowden, TA, Clark, BA, Sisly JF. Intraoperative serum and tissue activity of cefazolin and cefoxitin. Arch Surg 1985;120(7):829-32.

26. Michael KA, Henderson PL, Newman RB, Blackwelder EN, Caldwell RD. Impact of a pharmacist/physician cooperative target drug monitoring program on prophylactic antibiotic prescribing in obstetrics and gynecology. Hosp Pharm 1992;27(3):213-6.

27. Mallet HP, Njikam A, Scouflaire SM. Evaluation of prescription practices and of the rational use of medicines in Niger. Sante 2001;11(3):185-93.

28. Navarro S, Padilla CM. Indicadores estadísticos para la evaluación de protocolos asistenciales en oftalmología. Rev Cubana Oftalmol 2015;28(1):64-77.

29. Wright JD, Hassan K, Ananth CV, Herzog TJ, Lewin $\mathrm{SN}$, Burke WM, et al. Use of guideline-based antibiotic prophylaxis in women undergoing gynecologic surgery. Obstet Gynecol 2013;122(6):1145-53.

30. Von Gunten V, Reymond JP, Beney J. Clinical and economic outcomes of pharmaceutical services related to antibiotic use: a literature review. Pharm World Sci 2007;29(3):146-63. 